\title{
'Genetic heterogeneity' in HER2/neu testing by fluorescence in situ hybridization: a study of 2522 cases
}

\author{
Martin C Chang ${ }^{1,2,3}$, Janet I Malowany ${ }^{1,2,3}$, Julita Mazurkiewicz ${ }^{1}$ and Martha Wood ${ }^{1}$ \\ ${ }^{1}$ Department of Pathology and Laboratory Medicine, Mount Sinai Hospital, Toronto, ON, Canada and \\ ${ }^{2}$ Department of Laboratory Medicine and Pathobiology, University of Toronto, Toronto, ON, Canada
}

\begin{abstract}
Amplification for the ERBB2 oncogene encoding the HER2/neu protein (HER2) is of predictive and prognostic importance in breast carcinoma. Fluorescence in situ hybridization (FISH) is a widely accepted method for determining HER2 amplification status. A HER2-amplified tumor is defined as having a ratio of HER2 signals to chromosome 17 centromeric probe signals (HER2/CEP17 ratio) exceeding 2.2. However, the presence of scattered cells demonstrating HER2 amplification is of unclear significance. A 2009 panel guideline defined a tumor with 'genetic heterogeneity' as having at least $5 \%$ but fewer than $50 \%$ of (non-clustered) tumor nuclei with a ratio $>$ 2.2. The study objective was to examine the statistical distribution of breast tumors tested by FISH for HER2 amplification, after implementation of this 2009 guideline. We identified 2522 consecutive breast carcinoma cases (2009-2011) tested for HER2 amplification. All cases were tested by FISH using a standard clinical protocol, adhering to established guidelines. For each case, data on cell counts were retrieved electronically. Each tumor was compared with a theoretical normal distribution by quantile-quantile analysis. Of 2522 FISH tests for HER2, 1900 (75\%) were non-amplified, $394(16 \%)$ were amplified, and $228(9 \%)$ were HER2equivocal. A total of $666(26 \%)$ had 'genetic heterogeneity.' Among these 'genetically heterogeneous' cases, the ratio was non-amplified in $430(64.5 \%)$, amplified in $24(4 \%)$, and equivocal in $212(31.5 \%)$. The amplified subpopulation in 'genetically heterogeneous' tumors was larger if the overall ratio was close to 2.2. However, the percentage of nuclei $>\mathbf{2 . 2}$ in a 'genetically heterogeneous' tumor was not informative of the underlying tumor-cell distribution. We conclude that the proportion of HER2-amplified nuclei within a tumor does not contribute information independent of the actual HER2/CEP17 ratio. Reassessment of the definition of 'genetic heterogeneity' in HER2 testing is warranted.

Modern Pathology (2012) 25, 683-688; doi:10.1038/modpathol.2011.206; published online 27 January 2012
\end{abstract}

Keywords: breast carcinoma; genetic heterogeneity; HER2/neu; in situ hybridization

Human epidermal growth factor receptor 2 (HER2) is a membrane tyrosine kinase encoded by the ERBB2 oncogene. ${ }^{1}$ HER2 is commonly overexpressed or amplified in invasive breast carcinomas, a finding that correlates to high tumor aggressiveness and higher response rates to both chemotherapy (eg, doxorubicin) and targeted anti-HER2 therapy (eg, trastuzumab, lapatinib). ${ }^{1-5}$ Both immunohistochemistry and in situ hybridization are accepted methods

Correspondence: Dr MC Chang, MD, PhD, FRCPC, Department of Pathology and Laboratory Medicine, Mount Sinai Hospital, 600 University Avenue, 6-500, Toronto, ON M5G 1X5, Canada.

E-mail: mchang2@mtsinai.on.ca

${ }^{3}$ These two authors are principal authors.

Received 8 September 2011; revised 25 October 2011; accepted 25

October 2011; published online 27 January 2012 of determining clinical HER2 status. ${ }^{6-8}$ There is no universal agreement upon a 'gold-standard' method; however, in situ hybridization is considered to be reliable and reproducible when objective criteria are applied. ${ }^{8-10}$ In current practice, in situ hybridization is used either as a primary method of HER2 determination, ${ }^{10}$ or as an additional method to resolve cases that are equivocal by immunohistochemistry. ${ }^{9}$

Establishing a definition for HER2 gene amplification in breast carcinoma has been an ongoing challenge. HER2 determination by fluorescence in situ hybridization (FISH) requires the counting of HER2 probe signals and, in most cases, of a chromosome 17 centromeric probe (CEP17). At the time of the original trastuzumab trials, tumors with a HER2/CEP17 ratio of $>2.0$ by in situ hybridization were considered HER2-amplified. ${ }^{8}$ Further analysis 
showed that testing discordances arose up to $20 \%$ of the time, attributed to statistical variability near the clinical threshold. ${ }^{11}$ Under a widely accepted guideline, ${ }^{9}$ issued by a joint College of American Pathologists and American Society of Clinical Oncology (CAP/ ASCO) panel in 2007, an equivocal interval (HER2/ CEP17 ratio of 1.8-2.2) is used to address this source of variability.

Not addressed by the 2007 CAP/ASCO guideline is another important source of uncertainty: cases that appear to demonstrate intratumoral heterogeneity for HER2 gene amplification. These cases are challenging because they raise the possibility of a subpopulation of HER2-amplified tumor cells-a phenomenon thought to be uncommon but potentially significant. ${ }^{10,12-15}$ However, it is difficult to determine which tumors are heterogeneous, and therefore to determine the clinical significance of intratumoral heterogeneity. To facilitate the prospective collection of these data, the CAP/ASCO panel issued a supplemental guideline in 2009 to define tumors that are 'genetically heterogeneous. ${ }^{16}$ Based on expert opinion but not on clinical evidence, 'genetically heterogeneous' tumors were defined as those with at least $5 \%$ but fewer than $50 \%$ of nuclei having a HER2/CEP17 ratio $>2.2$. The objective of this study was to review the HER2 FISH testing data available since the implementation of the CAP/ ASCO 2009 guideline. Using a descriptive-statistical approach, we evaluate the extent to which the 2009 CAP/ASCO definition of 'genetic heterogeneity' identifies non-uniform tumor populations.

\section{Materials and methods}

\section{Clinical Case Selection}

Consecutive cases of breast carcinoma $(N=2522)$ tested for HER2 gene amplification by FISH, from April 2009 to June 2011 (inclusive), were identified in accordance with the procedures approved by the institutional research ethics board. These cases represent output from a regional reference center conducting both primary assessment (of biopsies) by FISH, and reflex-testing for (excisional) cases that were HER2-equivocal (or unsatisfactory) by immunohistochemistry. Because the objective of the study was to assess heterogeneity in gene amplification specifically, cases were not analyzed with respect to immunohistochemical staining.

\section{FISH Interpretation}

Clinical FISH processing and scoring at our institution follows a highly standardized protocol. Hybridization is performed using HER2/neu (nuc ish 17q11.2-q12) and CEP17 (nuc ish 17p11.1-q11.1) probes from Vysis (Des Plaines, IL, USA). All cases were scored by the same technologists (either JM, MW; or both in equivocal cases) with appropriate pathologist guidance, in accordance to CAP/ASCO guidelines. ${ }^{9,16}$ Images from all cases were reviewed by the signing pathologist to ensure representative scoring of the invasive component. All tumor areas on the slide were scanned to ensure capture of amplified subpopulations. Sporadic nuclei found to have single CEP17 signals were considered artifactually truncated, and not included in counting. Per guidelines, clusters of amplified cells were scored separately.

For both biopsies and excisions, at least 20 nuclei were counted (mean of 42, ranging from 20 to 120), with more nuclei counted in equivocal cases. As per the current ASCO/CAP guidelines, a HER2/CEP17 ratio was considered negative if $<1.8$; equivocal if $\geq 1.8$ but $\leq 2.2$; and positive if $>2.2 .{ }^{9}$ 'Genetic heterogeneity' was defined as having at least $5 \%$ but fewer than $50 \%$ of non-clustered infiltrative tumor cells with a ratio higher than $2.2{ }^{16}$

\section{Electronic Data Retrieval and Analysis}

Electronic laboratory worksheets were opened by an automated search, and the following data elements were retrieved: number of nuclei counted, mean number of HER2 and CEP17 signals, and the mean HER2/CEP17 ratio. In addition, the HER2/CEP17 ratio of each cell nucleus was retrieved, and quantilequantile (Q-Q) analysis was performed using an automated script (written in Python; http://www. python.org) as follows. A sorted rank-list of actual HER2/CEP17 values was retrieved from the laboratory worksheet. The mean HER2/CEP17 ratio and standard deviation of the actual data were determined and used to generate a matched sorted ranklist of theoretical values conforming to a normal distribution. To estimate the closeness-of-fit of the actual data to a normal distribution, least-squares regression analysis was performed on the paired sorted rank-lists, and the correlation coefficient $\left(R^{2}\right)$ was determined using standard statistical methods.

\section{Results}

\section{Prevalence of HER2 Amplification and 'Genetic Heterogeneity' $(N=\mathbf{2 5 2 2})$}

The HER2 amplification results of all cases evaluated, as defined by HER2/CEP17 ratio, are summarized in Table 1 (left). Of 2522 consecutive cases, $394(16 \%)$ were HER2-amplified (ratio $>2.2$ ), and $1900(75 \%)$ were HER2-non-amplified (ratio <1.8). The HER2/CEP17 ratio fell within the equivocal range (1.8-2.2) in 228 (9\%) cases.

As shown in Table 1 (right), 666 cases were classified as 'genetically heterogeneous' by the CAP/ASCO (2009) guideline (26\% of all cases). The majority of 'genetically heterogeneous' cases was HER2-nonamplified $(430 / 666,64.5 \%)$, and few were HER2amplified $(24 / 666,4 \%)$. Although cases classified as 
Table 1 Prevalence of HER2 gene amplification and 'genetic heterogeneity' $(N=2522)$

\begin{tabular}{lccc}
\hline HER2 amplification status (based on ratio) & \multicolumn{2}{c}{ Breakdown of 'genetically heterogeneous' cases } \\
\hline All cases & 2522 & 'Genetically heterogeneous' cases & $666(26 \%$ of all cases) \\
Amplified $(>2.2)$ & $394(16 \%)$ & Amplified $(>2.2)$ & $24^{\mathrm{b}}(4 \%)$ \\
Equivocal $(1.8-2.2)$ & $228(9 \%)$ & Equivocal $(1.8-2.2)$ & $212^{\mathrm{b}}(31.5 \%)$ \\
Non-amplified $(<1.8)$ & $1900(75 \%)$ & Non-amplified $(<1.8)$ & $430^{\mathrm{b}}(64.5 \%)$ \\
\hline
\end{tabular}

${ }^{\mathrm{a}}$ Genetically heterogeneous, defined as having $\geq 5 \%$ and $<50 \%$ amplified tumor cells (CAP/ASCO 2009).

${ }^{\mathrm{b}}$ Genetically heterogeneous cases comprise 24/394 (6\%) of amplified, 212/228 (93\%) of equivocal, and 430/1900 (23\%) of non-amplified cases.

'genetically heterogeneous' with an equivocal HER $2 / C E P 17$ ratio were in the minority $(212 / 666$, $31.5 \%)$, among all HER2-equivocal cases $(N=228)$, the vast majority were 'genetically heterogeneous' (212, 93\%). 'Genetically heterogeneous' cases comprised $24 / 430(6 \%)$ of amplified, 430/1900 (23\%) of non-amplified cases.

\section{'Genetic Heterogeneity' Increases with $\mathrm{HER} 2 / \mathrm{CEP17}$ Ratio as a Continuous Function}

Given that 'genetic heterogeneity' is defined by the percentage of tumor cells with a ratio $>2.2$. (ie, the percentage of amplified cells), the relation between this percentage and HER2/CEP17 ratio was evaluated graphically (Figure 1). Examination of the range of values shows that both HER2/CEP17 ratio and the percentage of amplified cells behave like continuous variables. The percentage of amplified cells was plotted on the ordinate against HER2/CEP17 ratio, showing that it increases as a continuous function. Within the 'genetically heterogeneous' range, this increase approximates a straight line.

The overall shape of the plot closely resembles the probability of any random variable-including those that are normally distributed-having a value above a given threshold. To illustrate this, the actual values in Figure 1 can be compared with the probability of representative normally distributed populations having values $>2.2$. The theoretical curve shown in Figure 1 (solid line) was constructed by taking a normal distribution for each data point having the same mean HER2/CEP17 ratio and a s.d. of 0.3 (comparable to the average standard deviation of the actual cases), and calculating the cumulative probability of HER2/CEP17 $>2.2$. As expected, when HER2/CEP17 ratio has a value of 2.2 (the amplification threshold), the percentage of amplified cells is $\sim 50 \%$. This finding does not depend on the actual distribution of HER2-amplified cells within the tumor (see below).

\section{Both Genetically Uniform and Non-Uniform Tumors may have a High Percentage of HER2-Amplified Cells}

Tumors in which HER2 is amplified according to a normal distribution (ie, genetically uniform tumors) could be identified either by inspecting a frequency

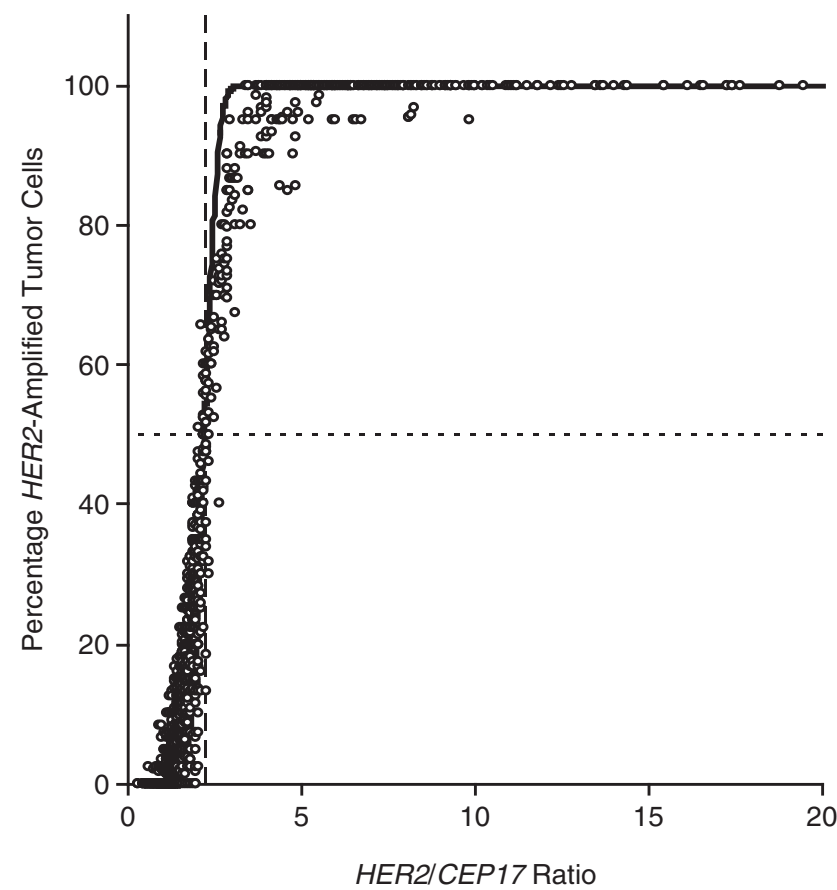

Figure 1 'Genetic heterogeneity' increases with HER2/CEP17 as a continuous function. The percentage of tumor cells with a HER2/ CEP17 ratio $>2.2$ is plotted against HER2/CEP17 ratio (hollow circles). For comparison, a theoretical curve (solid line) is plotted by calculating the probabilities of HER2/CEP17 $>2.2$ for a representative normally distributed population (mean $=$ HER2) $C E P 17$ ratio, s.d. $=0.3$ ). When HER2/CEP17 ratio has a value of 2.2 (the amplification threshold), the percentage of amplified cells is $\sim 50 \%$ (dotted lines) ( $N=2520$; for clarity two data points having HER2/CEP17 ratio $>20$ are omitted).

histogram of HER2/CEP17 ratio (Figure 2a), or by inspecting the plot of actual ranked values against a theoretical normal distribution matched with respect to both mean and s.d. (Q-Q plot, Figure 2b). As shown by the illustrative example in Figure 2b, genetically uniform tumors correspond to Q-Q plots that are well fitted by linear regression (ie, $R^{2}$ approaching 1.0).

A contrasting example of a genetically non-uniform tumor is illustrated in Figures 3 and 4. Such tumors could sometimes be identified visually during manual counting of HER2 and CEP17 signals under the fluorescence microscope (Figure 3). Within 

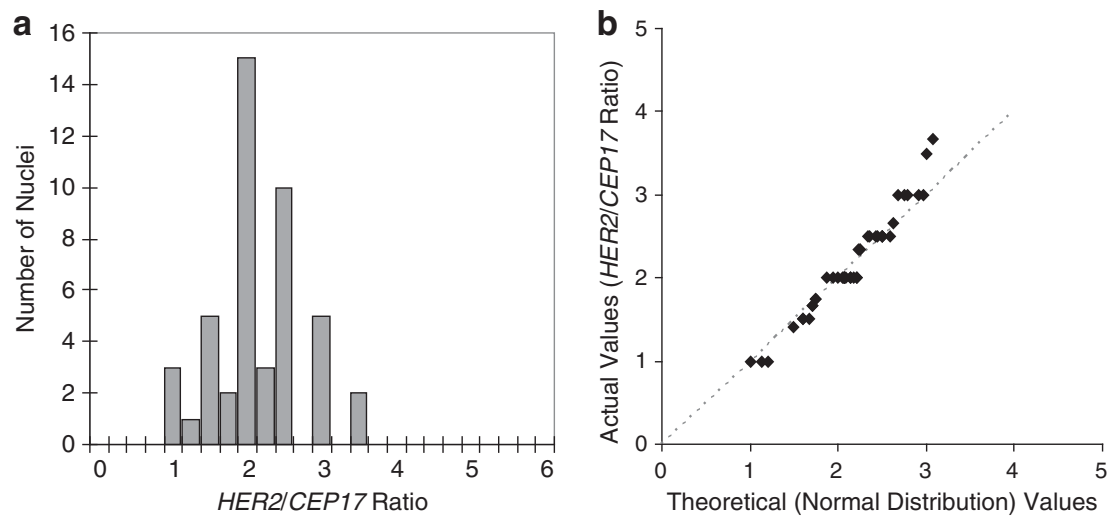

Figure 2 Example of a tumor with a uniform distribution having a high percentage of HER2-amplified cells. Example of a case with $H E R 2 / C E P 17$ ratio $=2.14$, with $42 \%$ of cells having a HER2/CEP17 ratio $>2.2$. As seen in the histogram (a), the HER2/CEP17 appears to be uniformly distributed around the mean. Quantile-quantile analysis (b) shows that the tumor population closely resembles a normal distribution $\left(R^{2}\right.$ of the actual-vs-theoretical plot $\left.=0.95\right)$.

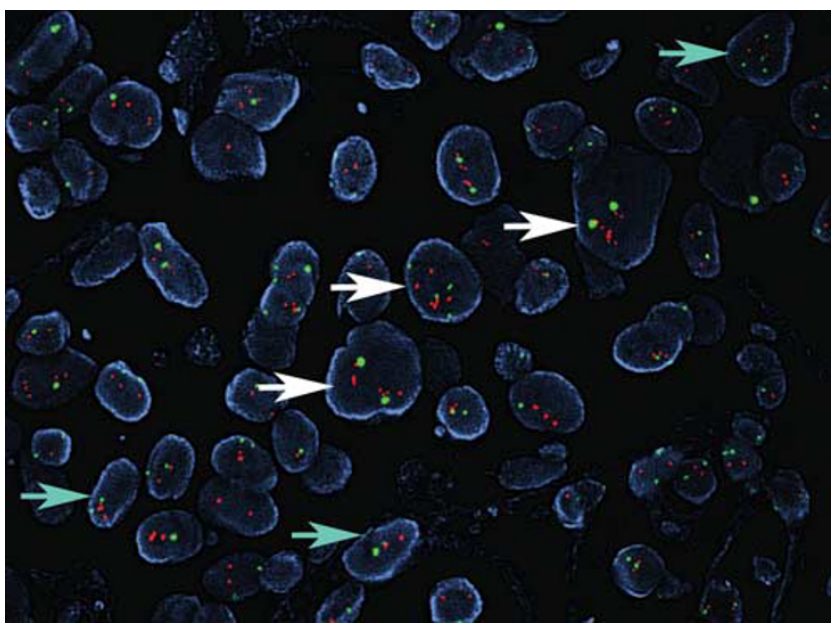

Figure 3 Microscopic appearance (FISH) of a tumor with a nonuniform population. Representative field (FISH microscopy, with orange HER2 signals, green CEP17 signals, and DAPI counterstain) of a case with $H E R 2 / C E P 17$ ratio $=1.90$, with $27 \%$ of cells having a HER2/CEP17 ratio $>2.2$. Both non-amplified (cyan arrows) and amplified (white arrows) nuclei are admixed (original magnification: $\times 1000$, oil immersion).

a single $\times 1000$ oil-immersion field, both HER2-nonamplified and (non-clustered) HER2-amplified cells can easily be identified, suggestive of a non-uniform population. As seen in the corresponding histogram (Figure 4a), scattered cells with a HER2/CEP17 ratio of two- or threefold the mean ratio were present within this tumor. The Q-Q plot shows a clear deviation from the diagonal line, by both visual inspection and linear regression analysis (Figure $4 \mathrm{~b}$ ).

Although these two examples illustrate tumors with different distributions, both would be scored as HER2-equivocal (ratio of 2.14 in the uniform tumor and 1.90 in the non-uniform tumor). Both tumors would also be classified as 'genetically heterogeneous' (the percentage of amplified cells was 42 and $27 \%$, respectively.)
The Percentage of HER2-Amplified Tumor Cells Is Not Informative of the Underlying Distribution

The correlation coefficient $\left(R^{2}\right)$ obtained by linear regression analysis of the Q-Q plot allows the comparison of tumor-cell distribution across different cases. For all 2522 cases, $R^{2}$ of the Q-Q plot was plotted against the percentage of amplified cells, to determine whether 'genetically heterogeneous' tumors correspond to genetically non-uniform tumors (Figure 5). There was no correlation between the $R^{2}$ value and the percentage of amplified cells.

\section{Discussion}

A review of 2522 consecutive cases tested by FISH shows that 'genetic heterogeneity' as defined by the 2009 CAP/ASCO guideline ${ }^{16}$ occurs in a significant minority $(26 \%)$ of all cases, and is not informative of non-uniformity within a tumor-cell population. Rather, the degree of 'genetic heterogeneity' increases as the overall HER2/CEP17 ratio increases to approach the 'cut point' of 2.2-as statistically expected for any continuous variable. Therefore, 'genetic heterogeneity' does not appear to contribute information about HER2 amplification independent of the HER2/ CEP17 ratio itself.

The purpose of the 2009 'genetic heterogeneity' definition was to address the possibility that HERamplified subpopulations of tumor cells may be significant for prognostic and predictive purposes. ${ }^{16}$ The 2009 CAP/ASCO guideline was based on expert opinion rather than clinical/pathologic evidence, and has generated some discussion. ${ }^{17-19}$ Nevertheless, the need to collect data on potential HER2amplified subpopulations was cited as a key factor in implementing this guideline. ${ }^{19}$ A large European audit performed by Bartlett et $a l^{20}$ linked to clinicaltrial data showed that the percentage of tumor cells with HER2/CEP17 ratios $>2.2$ does not identify cases with poor clinical outcome, and also that the 

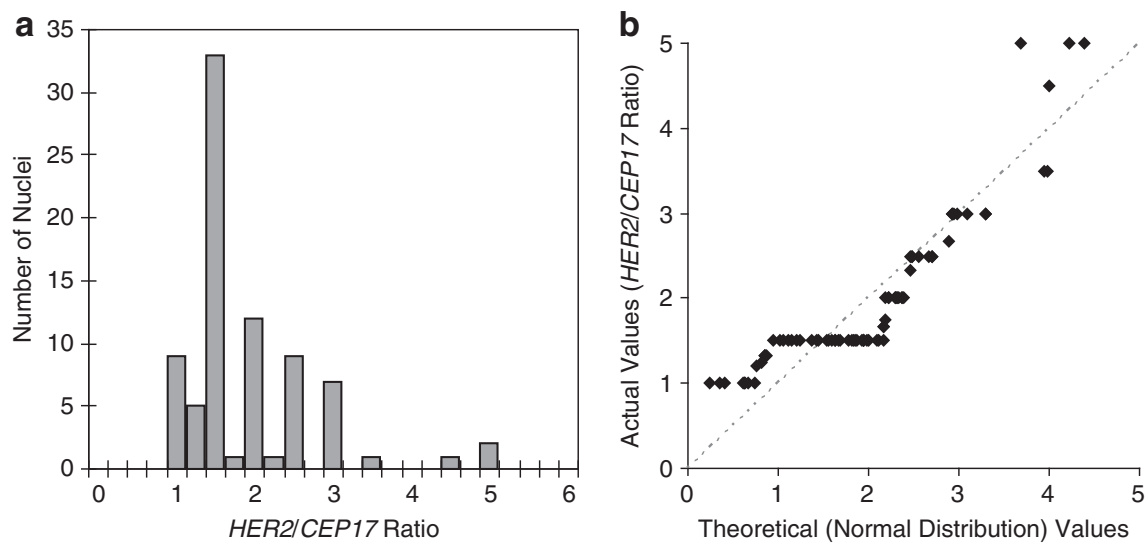

Figure 4 Example of a tumor with a non-uniform distribution, highlighted by Q-Q analysis. Example of a case (same as Figure 3) with HER2/CEP17 ratio $=1.90$, with $27 \%$ of cells having a HER2/CEP17 ratio $>2.2$. As seen in the histogram (a), some of the scattered amplified cells may represent a separate population. Quantile-quantile analysis (b) suggests a corresponding deviation from a normal distribution $\left(R^{2}\right.$ of the actual-vs-theoretical plot $\left.=0.84\right)$.

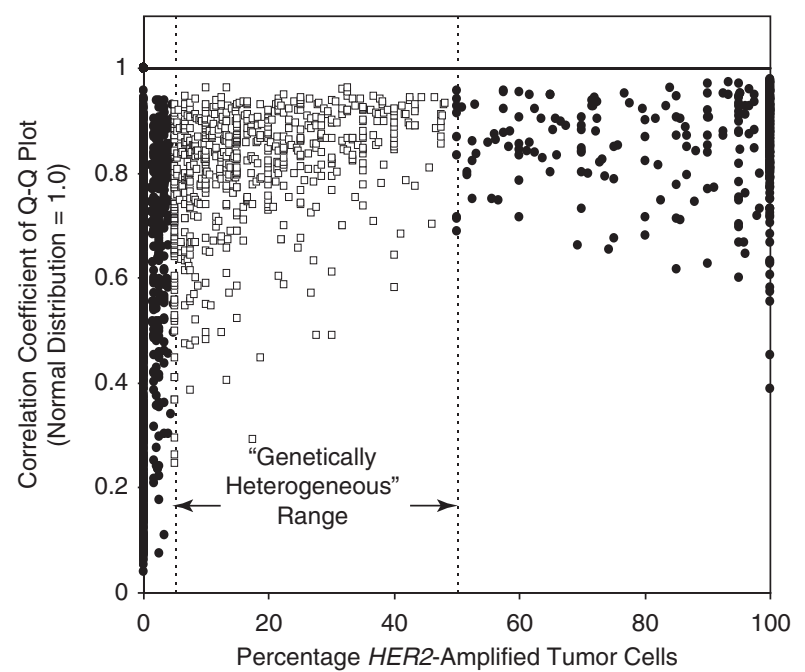

Figure 5 The percentage of HER2-amplified tumor cells is not informative of the underlying distribution. The closeness-of-fit to a normal distribution $\left(R^{2}=1\right)$, estimated by the $R^{2}$ values of $\mathrm{Q}-\mathrm{Q}$ plots, is plotted against the percentage of tumor cells with a HER2/CEP17 ratio $>2.2(N=2522)$. Cases defined as 'genetically heterogeneous' (between the dotted lines) are highlighted as hollow squares. There is no correlation between 'genetic heterogeneity' and tumor non-uniformity.

prevalence of 'genetic heterogeneity' is relatively high (up to $33 \%$ ). Although Bartlett et $a l^{20}$ found that a HER2-amplified-cell percentage of $30 \%$ may be clinically significant, it is unclear whether this significance is independent of the HER2/CEP17 ratio. The current study would suggest the contrary: that this apparent significance of a higher amplifiedcell percentage is a statistical artifact. The data therefore suggest that the 2009 'genetic heterogeneity' definition needs reassessment and modification.

Tumors tested by FISH may demonstrate a highly uniform population or one that is widely distributed with respect to HER2/CEP17 ratio. Neither the overall ratio nor the percentage of amplified cells can distinguish between these scenarios. It has been proposed that the presence of scattered amplified cells is an artifact of the technical and biologic aspects of using in situ hybridization in the detection of gene amplification. ${ }^{14,20}$ These include variability in cell sampling, completeness of nuclei, and intrinsic variability in tumor aneuploidy.

The 2009 ASCO/CAP guidelines recommend distinguishing between tumors with amplified clusters of cells from those with an admixture of cells. ${ }^{16}$ The current study does not address the presence of HER2-amplified cell clusters-it seems reasonable to strive for detection of discrete populations of amplified cells. Notwithstanding this recommendation, two problems remain: (1) how to identify tumors with truly heterogeneous HER2 amplification and (2) finding convincing evidence that this heterogeneity is of independent biologic significance beyond simple variability and artifact.

One proposal from Bartlett et $a l^{20}$ is to examine the average HER2/CEP17 ratio within distinct tumor areas (20-60 nuclei per area), and to define heterogeneous amplification as tumors containing both amplified and non-amplified areas. This approach, although correcting for artifacts detected in individual cells, does not take into account the statistical artifact inherent to defining a numerical threshold. That is, for any continuous variable, individual tumor cells will fall on both sides of any defined threshold. Similarly, one would expect individual tumor areas also to fall on both sides of this threshold. The current analysis shows that this effect is mathematically unrelated to intratumoral heterogeneity, but rather is predictably more pronounced as the average ratio approaches the threshold.

A truly rigorous definition of 'heterogeneity' should take into account the statistical distribution of tumor cells with respect to HER2/CEP17 ratio, which requires thorough sampling and counting. Such an approach is difficult and labor intensive. As automated methods of interpreting in situ 
hybridization based on image analysis become more widely adopted, ${ }^{21-23}$ it may become more feasible to determine the prevalence and significance of true tumor heterogeneity in the future. In the meantime, it must be acknowledged that the HER2/CEP17 ratio is a continuous variable, and that the definition of 'HER2 amplification' is, by necessity, dichotomous. There remains a lack of data addressing the effectiveness of treatment for tumors that do not fit well into the 'positive' or 'negative' categories. ${ }^{3,4,8}$

In conclusion, definitions of 'genetic heterogeneity' in HER2 FISH testing that depend only on the percentage/proportion of amplified cells are not informative of heterogeneity within a tumor population. Rather, the percentage of amplified cells varies with the HER2/CEP17 ratio in a predictable statistical manner. Current definitions of 'genetic heterogeneity' should be reassessed.

\section{Disclosure/conflict of interest}

Dr Martin C Chang has received research funding from Hoffman-LaRoche.

\section{References}

1 Navolanic PM, Steelman LS, McCubrey JA. EGFR family signaling and its association with breast cancer development and resistance to chemotherapy. Int J Oncol 2003;22:237-252.

2 Slamon DJ, Clark GM, Wong SG, et al. Human breast cancer: correlation of relapse and survival with amplification of the HER-2/neu oncogene. Science 1987;235:177-182.

3 Pritchard KI, Shepherd LE, O’Malley FP, et al. HER2 and responsiveness of breast cancer to adjuvant chemotherapy. N Engl J Med 2006;354:2103-2111.

4 Piccart-Gebhart MJ, Procter M, Leyland-Jones B, et al. Trastuzumab after adjuvant chemotherapy in HER2positive breast cancer. $N$ Engl J Med 2005;353: 1659-1672.

5 Jagiello-Gruszfeld A, Tjulandin S, Dobrovolskaya N, et al. A single-arm phase II trial of first-line paclitaxel in combination with lapatinib in HER2overexpressing metastatic breast cancer. Oncology 2010;79:129-135.

6 Jacobs TW, Gown AM, Yaziji H, et al. Comparison of fluorescence in situ hybridization and immunohistochemistry for the evaluation of HER-2/neu in breast cancer. J Clin Oncol 1999;17:1974-1982.

7 Jacobs TW, Gown AM, Yaziji H, et al. Specificity of HercepTest in determining HER-2/neu status of breast cancers using the United States Food and Drug Administration-approved scoring system. J Clin Oncol 1999;17:1983-1987.

8 Pauletti G, Dandekar S, Rong HM, et al. Assessment of methods for tissue-based detection of the HER-2/neu alteration in human breast cancer: a direct comparison of fluorescence in situ hybridization and immunohistochemistry. J Clin Oncol 2000;18:3651-3664.

9 Wolff AC, Hammond ME, Schwartz JN, et al. American Society of Clinical Oncology/College of American Pathologists guideline recommendations for human epidermal growth factor receptor 2 testing in breast cancer. J Clin Oncol 2007;25:118-145.

10 Tubbs RR, Hicks DG, Cook J, et al. Fluorescence in situ hybridization (FISH) as primary methodology for the assessment of HER2 Status in adenocarcinoma of the breast: a single institution experience. Diagn Mol Pathol 2007;16:207-210.

11 Dowsett M, Hanna WM, Kockx M, et al. Standardization of HER2 testing: results of an international proficiencytesting ring study. Mod Pathol 2007;20:584-591.

12 Glöckner S, Buurman H, Kleeberger W, et al. Marked intratumoral heterogeneity of c-myc and CyclinD1 but not of c-erbB2 amplification in breast cancer. Lab Invest 2002;82:1419-1427.

13 Shin SJ, Hyjek E, Early E, et al. Intratumoral heterogeneity of her-2/neu in invasive mammary carcinomas using fluorescence in-situ hybridization and tissue microarray. Int J Surg Pathol 2006;14:279-284.

14 Hanna W, Nofech-Mozes S, Kahn HJ. Intratumoral heterogeneity of HER2/neu in breast cancer-a rare event. Breast J 2007;13:122-129.

15 Sukov WR, Miller DV, Duek AC, et al. Benefit of adjuvant trastuzumab in breast cancer patients with focal HER2 amplified colonies: data from N9831 Intergroup Adjuvant Trial [abstract]. J Clin Oncol 2009;27: 25s(Suppl):520.

16 Vance GH, Barry TS, Bloom KJ, et al. Genetic heterogeneity in HER2 testing in breast cancer: panel summary and guidelines. Arch Pathol Lab Med 2009;133:611-612.

17 Hsu C-Y, Li AF, Yang C-F, et al. Proposal of modification for the definition of genetic heterogeneity in HER2 testing in breast cancer [letter to the editor]. Arch Pathol Lab Med 2010;134:162.

18 Albarracin C, Edgerton ME, Gilcrease MZ, et al. Is it too soon to start reporting HER2 genetic heterogeneity [letter to the editor]? Arch Pathol Lab Med 2010;134: 162-163.

19 Vance GH, Hicks DG. In reply [letter to the editor]. Arch Pathol Lab Med 2010;134:163.

20 Bartlett AI, Starcynski J, Robson T, et al. Heterogeneous HER2 gene amplification: impact on patient outcome and a clinically relevant definition. Am J Clin Pathol 2011;136:266-274.

21 Theodosiou Z, Kasampalidis IN, Karayannopoulou G, et al. Evaluation of FISH image analysis system on assessing HER2 amplification in breast carcinoma cases. Breast 2008;17:80-84.

22 Turashvili G, Leung S, Turbin D, et al. Inter-observer reproducibility of HER2 immunohistochemical assessment and concordance with fluorescent in situ hybridization (FISH): pathologist assessment compared to quantitative image analysis. BMC Cancer 2009;9:165.

23 Konsti J, Lundin J, Jumppanen M, et al. A publicdomain image processing tool for automated quantification of fluorescence in situ hybridisation signals. J Clin Pathol 2008;61:278-282. 